\title{
Is preparation for bronchoscopy optimal?
}

\author{
J. Pickles*, M. Jeffrey*, A. Datta\#, A.A. Jeffrey*
}

Is preparation for bronchoscopy optimal? J. Pickles, M. Jeffrey, A. Datta, A. A. Jeffrey. (C) ERS Journals Ltd 2003.

ABSTRACT: The results of a questionnaire survey, of the current preparation for and practice of diagnostic bronchoscopy in England and Wales, are reported in this paper. The British Thoracic Society (BTS) has recently published guidelines on bronchoscopy and these provide a consensus statement on the current evidence base. There is no specific guidance on drugs or techniques, although it is recommended that all patients should be offered sedation, except where there are contraindications.

In the present survey, there was a response rate of $76 \%$ (344 responses to 452 questionnaires) and the median number of bronchoscopies performed per session was 5 (interquartile range 4-6). Most operators use lignocaine gel to the nose (65\%), spray to the throat $(70 \%)$, followed by the "spray as you go" method $(84 \%)$, recommended by the BTS. Atropine is routinely used by $13 \%$ contrary to the guidelines and despite concerns about its side-effects. Most operators use sedation with midazolam $(85 \%)$ or a wide variety of combinations of sedative, analgesic, and anaesthetic agents (27\%), and $27 \%$ perform unsedated bronchoscopies, with only $0.1 \%$ routinely performing unsedated bronchoscopies. A total $251(77 \%)$ responders stated they assessed adequacy of sedation, with most using patient observation alone $(149(46 \%))$. Only three operators assessed sedation using a formal sedation score. Thus, most centres routinely perform sedated bronchoscopies and the systematic level of monitoring is poor.

The current controversies about sedation and safe sedation practice are discussed. There is a need for more evidence to allow more specific guidance to be produced in this difficult area.

Eur Respir J 2003; 22: 203-206.
*Chest Clinic, Northampton General Hospital, Northampton and ${ }^{\#}$ Respiratory Centre, Portsmouth Hospitals National Health Service (NHS) Trust, Portsmouth, UK.

Correspondence: J. Pickles

Chest Clinic

Northampton General Hospital

Northampton

NN1 5BD

UK

Fax: 441604544858

E-mail: jpickles@doctors.org.uk

Keywords: Anxiolytic

flexible fibreoptic bronchoscopy

safe sedation

Received: December 192002

Accepted after revision: April 172003
The bronchoscopic technique is not standardised and the ideal preparation for diagnostic bronchoscopy is not known. The current British Thoracic Society (BTS) guidelines [1] provide a consensus statement on the current evidence base without specific guidance on drugs or techniques and without defining methods of sedation. The guidelines recommend offering sedation to all undergoing diagnostic flexible fibreoptic bronchoscopy, except where there are contraindications [1]. The aim is to achieve good patient tolerance, comfort and cooperation whilst reducing complications. The issues of sedation are controversial. If a centre has experience of performing unsedated diagnostic flexible fibreoptic bronchoscopy, it is reported that patient cooperation is not improved with sedation [2]. Furthermore there is worrying evidence that insufficient monitoring of sedated patients occurs, potentially placing patients at risk [3]. One-half of deaths reported are related to sedation [4]. It is unclear what constitutes optimum sedation. A Report by an Intercollegiate Working Party suggests safety measures for sedation undertaken by an operator who is not anaesthetics-trained (table 1) [5]. As bronchoscopy may be performed safely without sedation, when it is used there is emphasis on its safe administration.

In this survey, the authors aimed to obtain a "snap shot" view of the current national flexible fibreoptic practice for bronchoscopy in adults and the sedation, analgesia and anaesthesia used therein.
Methods

Questionnaires were faxed to respiratory consultants listed in the BTS directory [6] after a telephone call to the department secretary. A follow-up call was made $2-4$ weeks after the fax. The questionnaire contained mainly tick-box questions on a single side of A4 paper that would take individual a few minutes to complete. Questions focused on the patient population covered, numbers of consultants and bronchoscopies undertaken, the types of topical anaesthetic used for the nose, throat, vocal cords and bronchi, and sedative or anaesthetic use. Finally, the operator was asked to describe how they monitored the effects of sedation if this was used.

Each respondent had their date of appointment to their consultant physician post checked in the BTS directory and the length of time in the post used as a surrogate for length of experience.

Descriptive statistics and Chi-squared tests comparing two groups of operators (divided by their experience) were performed.

\section{Results}

Four-hundred and fifty-two questionnaires were faxed to consultants at 234 hospitals and 344 responses were received (response rate $76 \%$ ). The median number of consultants per 
Table 1.-Intercollegiate Working Party: safe sedation guidelines

Verbal contact should be maintained at all times (i.e. "conscious sedation")

When the intravenous route is used, intravenous access should be present at all times

Avoid polypharmacy

When using an opioid/sedative combination the opioid should be administered first

There should be a defined and trained person who records monitoring

Oxygen and devices to deliver it should be available

Patient trolleys should be equipped to tip the head down

Resuscitation equipment should be available

There should be defined methods of sedation

The operator should have received training in sedation

Table 2.-Use of topical anaesthetic to the nose and throat

\begin{tabular}{lc}
\hline Topical agent used & Number (\%) using topical agent \\
\hline Topical cocaine & $4(1)$ \\
Lignocaine & $148(43)$ \\
$\quad$ Spray to nose & $225(65)$ \\
Gel to nose & $242(70)$ \\
Spray to throat & $25(7)$ \\
Nebulised & $6(2)$ \\
Amethocaine Lozenges & \\
\hline
\end{tabular}

hospital unit was three (range 1-20). The median population served per consultant was 116,667 people with an interquartile (IQR) range of $90,000-150,000$ people. The median number of bronchoscopies performed per session was five (IQR 4-6). The reported numbers using topical anaesthetics to the nose and throat are shown in table 2 . The reported numbers using topical anaesthetics to the vocal cords and bronchi are shown in table 3.

\section{Topical anaesthetic}

The majority of respondents used lignocaine spray to the throat with gel to the nose and some also used an additional spray to the nose. Four per cent lignocaine was most commonly used for vocal-cord anaesthesia and 2\% lignocaine for the bronchi. From the responses received, 16\% did not report

Table 3.-Use of topical anaesthetic to the vocal cords and bronchi

\begin{tabular}{lc}
\hline Topical agent used & Number (\%) using topical agent \\
\hline Lignocaine & \\
Trans-crycoid & $49(14)$ \\
$4 \%$ to cords & $185(54)$ \\
$2 \%$ to cords & $127(37)$ \\
$4 \%$ to bronchi & $44(13)$ \\
$2 \%$ to bronchi & $245(71)$ \\
\hline
\end{tabular}

giving 2-4\% lignocaine to the bronchi. A few operators omitted nasal anaesthesia as they passed the bronchoscope orally.

\section{Atropine}

Sixty-one of 306 consultants that replied used atropine. Only 41 used this routinely, with the other operators using it in patients with asthma $(n=5)$, bradycardias $(n=5)$, history of vasovagal episode $(n=1)$, for unspecified reasons $(n=5)$ and when excess secretions were present $(n=4)$. One operator routinely used glycopyrrolate.

\section{Sedation}

Most performed bronchoscopies using sedation with midazolam (table 4). Overall, 93 (27\%) performed unsedated bronchoscopies, with the majority of operators resorting to this because of respiratory failure and/or other factors, such as patient age, frailty and comorbidity. A significant minority $(n=29$ or $8.4 \%$ ) stated that they discussed the options of sedation with the patient. Of these, 28 stated that they used sedation routinely. Of the 18 who routinely performed unsedated bronchoscopy, only one stated that they offered the patient an option on sedation; they reported that more than one-half the patients opted not to have sedation.

Where a combination of sedatives and systemic analgesics was used, the commonest combination was midazolam plus the addition of alfentanil/fentanyl $(n=48)$, with the opioid added, in some cases, when the operator was concerned about cough $(n=4)$ and midazolam when the operator was concerned about patient anxiety $(n=14)$. The combinations of systemic medications used to achieve sedation and/or anxiolysis are shown in table 5 .

When sedation was employed, only three operators used formal sedation scores. Of the 326 who routinely sedate, 251 $(77 \%)$ indicated how they assessed adequacy of sedation. Onehundred and forty-nine (46\%) operators used patient observation alone, $46(14 \%)$ used measurement of vital signs alone and $56(17 \%)$ used a combination of patient observation and measurement. Table 6 shows the number of respondents using observations and measurements described in free-text (consultants were asked open-ended questions and were free to put their own comments and interpretation of the question down in writing and no word limit was set).

It is clear from the operators' comments that the level of sedation aimed for varies greatly from achieving amnesia, to anxiolysis, and to inducing sleep. In addition, the methods used to achieve the different aims were highly variable, with some operators titrating doses and others using a fixed predetermined dose that they felt produced adequate sedation in all. Some examples of the free-text descriptions of how operators assess levels of sedation are shown in table 7 .

\section{Sedation and operator experience}

There is no link between the number of bronchoscopies performed per session and the operators' willingness to use unsedated bronchoscopy.

Table 4.-Sedation practices

\begin{tabular}{lccccc}
\hline & Routine & Often & Sometimes & Rarely & Respiratory failure \\
\hline None & 18 & 1 & 7 & 6 & 61 \\
Midazolam & 269 & 5 & 8 & 11 & 0 \\
Other sedatives & 77 & 1 & 6 & 9 & 0 \\
\hline
\end{tabular}


Table 5. - Summary of other sedative practices

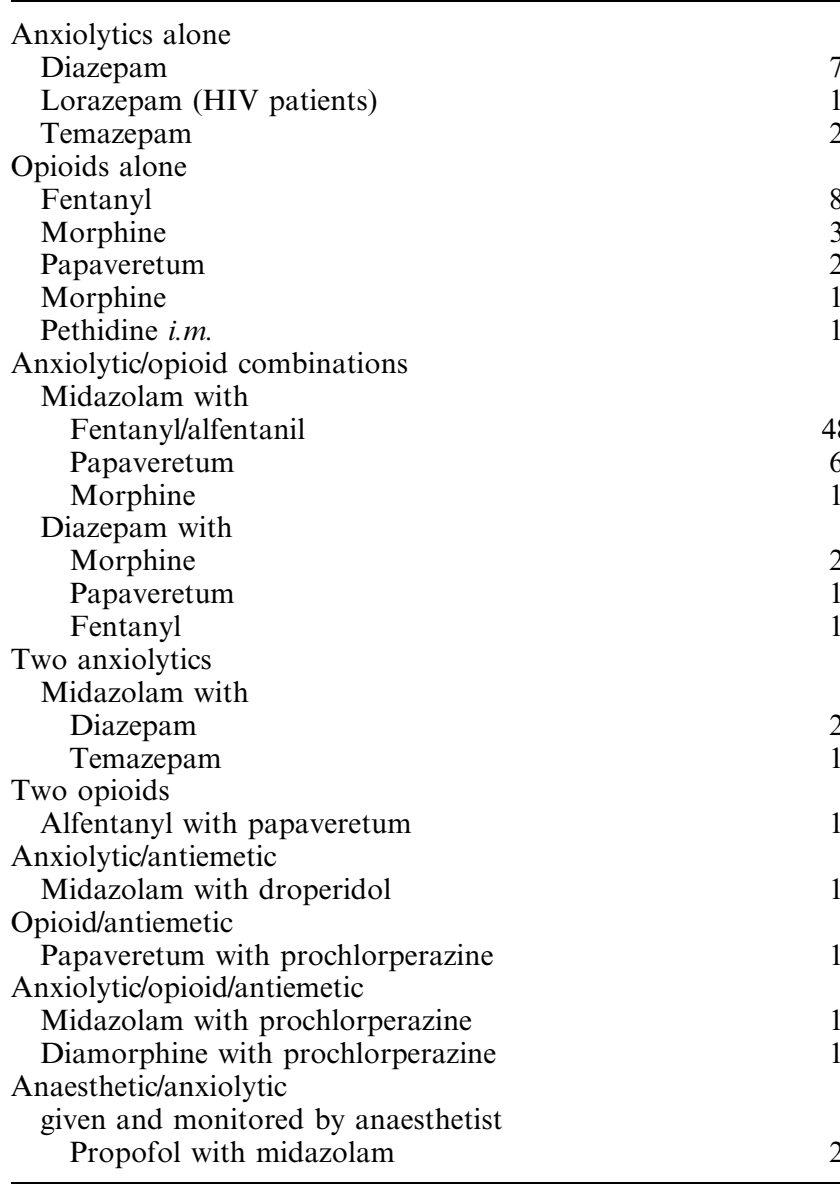

Data are presented as number of operators. HIV: human immunodeficiency virus; i.m.: intramuscular.

There is no relationship between operator experience and topical anaesthetic use. Respondents appointed to their post within the last $10-15 \mathrm{yrs}$, corresponding to a period when fibreoptic bronchoscopy training became routine, are more likely (although not statistically so) to perform unsedated bronchoscopy than those appointed earlier.

Table 6.-Assessment of sedation

Operator $n$

\begin{tabular}{lc}
\hline Measurements & \\
Oxygen saturations & 98 \\
Cardiac frequency & 21 \\
Blood pressure & 4 \\
Respiratory rate & 27 \\
Vital signs & 1 \\
Patient observation & \\
Patient response & 57 \\
Verbal response & 27 \\
Eyelid flutter & 16 \\
Clinical acumen/experience & 60 \\
Tolerance/comfort/compliance & 45 \\
Conscious level/rousable/drowsiness & 50 \\
Cough & 2 \\
AVPU score & 1
\end{tabular}

AVPU score: awake, response to verbal command and pain, unresponsive. The numbers of operators making measurements and observations are shown. These numbers are not mutually exclusive. Thus, an individual operator uses a unique combination of individual measurements and observations to assess sedation.
Table 7.-Operators' assessment of adequacy of sedation

Monitoring of patient observations is adequate as patients are so lightly sedated no judgement on sedation is required

We are more interested in the level of oxygen saturations than the level of sleepiness

Aim for amnesia not sedation

Watch patients' response to topical lignocaine/bronchoscopy insertion/verbal commands after sedation is given

Induce relaxation without sleep

Titrate to drowsiness and slurred speech

If anxious, may sedate to sleepy rather than wakeful state

Ensure patient awake enough to talk and respond but sleepy enough to allow me to get on with it

Assess for alertness at the end of the procedure

Ask the patient if they are happy with the level of sedation prior to starting the procedure

Midazolam is by far the commonest sedative used. Operators using other forms of sedation and atropine are more likely to have been appointed to their post for $>10$ yrs ( $\mathrm{p}=0.001$ and $\mathrm{p}=0.006$, respectively, with Chi-squared testing).

(1)

For topical anaesthesia to the upper airways, the BTS guidelines suggest an optimum combination of lignocaine gel to the nose followed by the "spray as you go" method using aerosolised lignocaine. These methods were used in 65 and $70 \%$ of cases, respectively. In a small number of cases $(43 \%)$, lignocaine spray to the nose was used in addition to gel. The guidelines are based on reported patient preference for nasal gel rather than nasal spray. One study suggests patients find the transcricoid route more pleasant than the "spray as you go" method and, as a result, less lignocaine is required [1], thereby reducing the likelihood of exceeding the recommended dose, which has been shown to be a common occurrence $[8,9]$. The present results for topical anaesthesia are similar to those of other reports [7-9].

The BTS does not advise the routine use of atropine because of side-effects (bradycardia, tachycardia, palpitations, arrythmias, loss of pupillary accommodation, photophobia, dry mouth, flushing, confusion in the elderly, nausea, vomiting and giddiness). However, no advice is given on when its use might be justified. Nevertheless, atropine continues to be used by $20 \%$ and $13 \%$ use atropine routinely. These figures compare favourably with a previous survey, in which $68.6 \%$ of units were using atropine [3], and are comparable to more recent studies [7-9]. In this survey, 81\% (279) routinely sedated patients for bronchoscopies with benzodiazepines and a further 19\% (67) used a variety of preparations (table 5).

There are several studies indicating improved patient tolerance with benzodiazepines. However, these studies used high doses of midazolam or aimed to achieve levels of sedation that were deeper than the recommended "conscious sedation" and should therefore warrant anaesthetic presence [5]. Williams et al. [10] sedated 123 patients to "light sleep", with doses of midazolam ranging from $5-42.5 \mathrm{mg}$ and continuous supplemental oxygen of $3 \mathrm{~L} \cdot \mathrm{min}^{-1}$. Two patients had prolonged desaturation requiring flumazenil and 16 
had transient desaturations that were severe enough for 13 patients to require flumazenil because of lack of monitoring facilities. Six patients required admission and one required intubation. Patients had a recovery stay of $2.5 \mathrm{~h}$ [10]. In a later study, WILliams and BowIE [11] analysed data over 2 yrs, using the same sedation technique. Twenty-eight of 274 patients $(10 \%)$ required flumazenil and there were 11 admissions (4\%). The authors concluded that high patient acceptability correlated with amnesia. MAGUIRE et al. [12] used diazepam to produce an unresponsive drowsiness, which is deeper than the recommended "conscious sedation", and showed improved patient tolerance over the first $24 \mathrm{~h}$ but a prolonged recovery period. PUTINATI et al. [4] sedated to The Mental Alertness and Drowsiness Index 3 (Drowsy) and showed better tolerance to bronchoscopy with few adverse effects when patients were questioned $3 \mathrm{~h}$ after the procedure, at a time when amnesia is likely to occur.

Some groups have suggested that unsedated bronchoscopies are as tolerable for the patient without the risk of sedation. HATTON et al. [13] questioned the use of routine sedation in bronchoscopy when they failed to demonstrate a difference in patient tolerance with either opioid or anxiolytic. However, their study was criticised [14] as they did not record sedation level ("light sedation"). MALTIAs et al. [2] performed a double-blind placebo-controlled trial in 100 patients in a centre that normally performs unsedated bronchoscopy and could not demonstrate improved patient tolerance, comfort and cooperation with lorazepam. They demonstrated an amnesic effect, which was greatest at $24 \mathrm{~h}$, and this was associated with reported improved tolerability.

The present survey shows that there are wide variations in the level of sedation aimed for (table 7) and the level of sedation achieved in some cases would warrant anaesthetic cover [5]. There is also variation in the types of agents used, confirming other reports [7-9], and how they are administered. Despite the enormously different preparations used, there does appear to be a shift from using opiates by operators appointed $>10$ yrs ago to using midazolam in those more recently appointed. This appears to be borne out by previous studies, in which, in $1986,78 \%$ of operators used opiates alone and in 2001, 63\% used midazolam alone [7, 9].

Monitoring was also highly variable, confirming previous reports [3, 8, 9]. In $205(63 \%)$ cases, patient observation by the operator or assistant was performed. However, patient observation included several aspects of their clinical state (table 6) and did not always include the "verbal contact" recommended by the recent safe sedation guidelines [5]. This is a cause for concern, particularly as it has been recently shown that some patients are not fasted for the recommended period [9] and the use of two or more agents, such as benzodiazepines and opioids, by some operators, increases the risk of cardiorespiratory depression. There is currently no routine monitoring of complications.

Overall, the present survey has shown that the majority of bronchoscopists use topical nasal gel and lignocaine. There are wide variations in bronchoscopy preparations from unsedated to a combination of sedatives, opioids and antiemetics used. Most centres routinely perform sedated bronchoscopies and the level of monitoring is poor. The ideal sedative regime is not known. If high doses of sedation are used, this poses a risk to patient safety with high complication rates [4, 10-12], although sedation may not affect mortality [9]. If low doses of sedation are used, there is no improvement in patient tolerance compared to unsedated patients. In centres that routinely practice unsedated bronchoscopies, there is no difference in patient tolerance, but amnesia is achieved with sedation [2]. Unsedated bronchoscopies may avoid complications, reduce the time patients spend in hospital, and enable the results to be discussed immediately with the patient. Reports suggest that improved explanation to include the sensations patients might experience [15] and improved topical anaesthesia and analgesia [16] can reduce fear and pain [17].

Some units routinely perform unsedated bronchoscopy. Anecdotally, patients tolerate the procedure well with minimal increases in cardiac frequency and many watch the procedure live on video. If they tolerate the procedure well, is there a justification for routinely inducing amnesia? There is a need for better evidence on best practice and clear implementable guidelines.

\section{References}

1. British Thoracic Society Guidelines on Diagnostic Flexible Bronchoscopy. Thorax 2001; 56: Suppl. 1, i1-i21.

2. Maltias F, Laberge F, Laviolette $M$. A randomised, double-blind, placebo-controlled study of lorazepam as premedication for bronchoscopy. Chest 1996; 109: 11951198.

3. Honeybourne D, Neumann CS. An audit of bronchoscopy practice in the United Kingdom: a survey of adherence to national guidelines. Thorax 1997; 52: 709-713.

4. Putinati S, Ballerin L, Corbetta L, Trevisani L, Potena A. Patient satisfaction with conscious sedation for bronchoscopy. Chest 1999; 115: 1437-1440.

5. Implementing and ensuring safe sedation practice for healthcare procedures in adults. Report of an Intercollegiate Working Party chaired by The Royal College of Anaesthetists. The Academy of Medical Royal Colleges, London, 2001; pp. 1-16.

6. BTS. The Directory of Training Posts and Services in Adult and Paediatric Respiratory Medicine. 7th Edn. London, Hawker Publications, 2002.

7. McLean AN, Semple PDA, Franklin DH, Petrie G, Millar EA, Douglas JG. The Scottish multi-centre prospective study of bronchoscopy for bronchial carcinoma and suggested audit standards. Respir Med 1998; 92: 1110-1115.

8. Simpson FG, Arnold AG, Purvis A, Belfield PW, Muers MF, Cooke NJ. Postal survey of bronchoscopy practice by physicians in the United Kingdom. Thorax 1986; 41: 311-317.

9. Smith CM, Stead RJ. Survey of flexible fibreoptic bronchoscopy in the United Kingdom. Eur Respir $J$ 2002; 19: $458-463$.

10. Williams TJ, Nicoulet I, Coleman E, McAlaney C. Safety and patient acceptability of intravenous midazolam for fibreoptic bronchoscopy. Respir Med 1994; 88: 305-307.

11. Williams TJ, Bowie PE. Midazolam sedation to produce complete amnesia for bronchoscopy: 2 years' experience at a district general hospital. Respir Med 1999; 93: 361-365.

12. Maguire GP, Rubinfeld AR, Trembath PW, Pain MCF. Patients prefer sedation for fibreoptic bronchoscopy. Respirology 1998; 3: 81-85.

13. Hatton MQF, Allen MB, Vathenen AS, Mellor E, Cooke NJ. Does sedation help in fibreoptic bronchoscopy? BMJ 1994; 309: 1206-1207.

14. Parker MRJ, Day CJE, Coote AH. Sedation in fibreoptic bronchoscopy (letter). BMJ 1995; 310: 872.

15. Poi PJH, Chuah SY, Srinivas P, Liam CK. Common fears of patients undergoing bronchoscopy. Eur Respir J 1998; 11: $1147-1149$.

16. Diette GB, White JR, Terry P, Jenckes M, Wise RA, Rubin HR. Quality assessment through patient self-report of symptoms prefibreoptic and postfibreoptic bronchoscopy. Chest 1998; 114: 1446-1453.

17. Lechtzin N, Rubin HR, Jenkes M, White P, Zhou LM, Thompson DA. Predictors of pain control in patients undergoing flexible bronchoscopy. Am J Respir Crit Care Med 2000; 162: 440-445. 